\title{
Simultaneous Screening of Multiple Bacterial tRNA Synthetases Using an Escherichia coli S30-Based Transcription and Translation Assay
}

\author{
Michael Dermyer, Scott C. Wise, * Timothy Braden, and Tod P. Holler
}

\begin{abstract}
The search for novel antibiotics to combat the growing threat of resistance has led researchers to screen libraries with coupled transcription and translation systems. In these systems, a bacterial cell lysate supplies the proteins necessary for transcription and translation, a plasmid encoding a reporter protein is added as a template, and a complex mixture of amino acids and cofactors is added to supply building blocks and energy to the assay. Firefly luciferase is typically used as the reporter protein in high-throughput screens because the luminescent signal is strong and, since bacterial lysates contain no luciferase, the background is negligible. The typical coupled transcription and translation assay is sensitive to inhibitors of RNA polymerase and to compounds that bind tightly to the ribosome. We have found a way to increase the information content of the screen by making the assay more sensitive to inhibitors of tRNA synthetases. Restricting the concentration of amino acids added to the reaction mixture allows the simultaneous screening of multiple tRNA synthetase enzymes along with the classic transcription and translation targets. In addition, this assay can be used as a convenient way to determine if an antibacterial compound of unknown mechanism inhibits translation through inhibition of a tRNA synthetase, and to identify which synthetase is the target.
\end{abstract}

\section{Introduction}

$\mathbf{T}_{\mathrm{r} \sin }$ HE GROWING NUMBER of bacterial infections that are resistant to one or more antibiotics has stimulated the search for novel chemical entities that are capable of addressing these infections. Several approaches have been employed to discover novel antibacterials, including the rescreening of pathways known to be susceptible to intervention with orally available antibiotics. Transcription and translation are among these pathways because of the significant number of known inhibitors ${ }^{1}$ and the availability of well-established, robust assays based upon the synthesis of luciferase in Escherichia coli S30 lysates. $^{2}$ These assays have been extended to lysates of Gram-positive organisms ${ }^{3}$ and used to discover novel chemical entities that target RNA polymerase or the ribosome ${ }^{4}$ but they are not generally sensitive, at normal screening concentrations, to known inhibitors of tRNA synthetases, particularly those known to be competitive with the cognate amino acid (vide infra).

Many tRNA synthetases can be considered good targets for antibacterial discovery because they are broadly conserved, essential for growth, and distinct enough from their human orthologs to anticipate the discovery of selective inhibitors. ${ }^{5}$ The novel methionyl tRNA synthetase inhibitor REP8839, for example, is being developed as a topical antibiotic. ${ }^{6}$ The tRNA synthetases have typically been screened separately using radiolabeled amino acid substrates, with the resulting acylated tRNAs isolated by precipitation and filtration, or detected using scintillation

Pfizer Global Research and Development, Michigan Laboratories, Arbor, MI.

*Present address: Deciphera Pharmaceuticals, Lawrence, KS.

ABBREVIATION: $\mathrm{IC}_{50}, 50 \%$ inhibitory concentration. 
proximity assay technology. ${ }^{7}$ The leads developed from these screens are typically inhibitors of aminoacyladenylate formation, and competitive with the cognate amino acid. They lose considerable potency as the concentration of cognate amino acid is raised manyfold above the enzyme's $K_{\mathrm{m}}$ for this substrate. We considered the possibility that lysate-based transcription and translation assays are insensitive to inhibitors of tRNA synthetases because they are routinely supplemented with high concentrations (up to $1 \mathrm{~m} M$ ) of their cognate amino acids. We investigated the ability of $E$. coli $\mathrm{S} 30$ lysates to synthesize firefly luciferase when the amount of supplementing amino acids added to the assay was restricted, and used this information to design an HTS assay that should be sensitive to inhibitors of all the tRNA synthetases except arginine.

In addition to screening of selected targets and pathways, whole cell screening of both natural products and synthetic materials has continued to be a source of novel antibacterial compounds. A key step in developing these discoveries is defining the molecular target of the novel agent. While traditional macromolecular synthesis assays and in vitro transcription and translation assays can narrow the search, there has been no convenient way to test whether or not the target might be a tRNA synthetase and which of the tRNA synthetases is the actual target. The assay scheme presented here fills this niche.

\section{Materials and Methods}

E. coli S30 lysates

Lysates were either purchased commercially (Promega Corp., Madison, WI) or prepared in-house using standard procedures. $^{3}$

\section{E. coli transcription and translation assay (luciferase)}

The assay was done in both 96- and 1,536-well formats with a total assay volume of $35 \mu \mathrm{l}$ and $1.5 \mu \mathrm{l}$, respectively. The 96-well assay was performed in a solid black plate (Corning Inc., Acton, MA), and $17 \mu \mathrm{l}$ of enzyme mix was added, containing $3.4 \mu \mathrm{l}$ of S30 extract (Promega Corp.), $2.3 \mu$ of TMK Buffer (10 mM Tris$\mathrm{HCl}$ [pH 8.0], $14 \mathrm{~m} M$ magnesium acetate, $60 \mathrm{~m} M \mathrm{KCl}$, and $1 \mathrm{~m} M$ dithiothreitol), and $11.3 \mu \mathrm{l}$ of diethyl pyrocarbonate-treated water. One microliter of compound in dimethyl sulfoxide was added, and the assay was started with $17 \mu \mathrm{l}$ of an initiation mix consisting of $9 \mu \mathrm{l}$ of S30 Pre-Mix (Promega Corp.), $2 \mu$ l of amino acid supplement as described in individual experiments, $2.3 \mu \mathrm{l}$ of TMK Buffer, and $3.7 \mu \mathrm{l}$ of $0.27 \mathrm{mg} / \mathrm{mL}$ pBESTluc Plasmid (Promega Corp.). The assay was left at room temperature for $1 \mathrm{~h}$, quenched with $35 \mu \mathrm{l}$ of Luc-Lite Plus (Perkin-Elmer Inc., Wellesley, MA), and read using the default luminescence mode on a Analyst HT microplate reader (Molecular Devices Corp., Sunnyvale, CA). The components for the 1,536-well assay were exactly the same as for the 96-well assay, just miniaturized to a 1.5$\mu \mathrm{l}$ volume. For the 1,536-well assay, $30 \mathrm{nl}$ of compound was spotted onto a black clear-bottom low-base plate (Aurora Discovery, San Diego, CA) using a Piezo Sample Distribution Robot (Aurora Discovery). Seven hundred fifty nanoliters of enzyme mix and initiation mix was added with a Flying Reagent Dispenser (Aurora Discovery). The assay was left to incubate in the dark at $30^{\circ} \mathrm{C}$ for $4 \mathrm{~h}$, then quenched with $1.5 \mu \mathrm{l}$ of Luc-Lite Plus, and read using an Acquest microplate reader (Molecular Devices Corp.).

\section{Results}

Response of an E. coli transcription and translation assay to lowered amino acid concentration

The typical commercially available transcription and translation assay is assembled from four components: (1) the S30 fraction that contains the ribosomes and other polypeptides necessary for transcription and translation, (2) a "premix" that contains nucleotides and small molecule cofactors necessary to sustain the process, (3) an amino acid mixture that is either complete, or lacks certain amino acids to allow the user to introduce a radiolabel into the assay, and (4) a plasmid containing the reporter gene. While a variety of reporters have been used for this assay, firefly luciferase is typically used in HTS campaigns.

High-activity S30 fraction is typically prepared in a two-step procedure. The first step involves cell lysis and centrifugation, and the second step involves an exhaustive translation reaction meant to remove endogenous mRNAs. After this second step the $\mathrm{S} 30$ lysate is dialyzed to remove small molecules. From this, one might suspect that the residual amino acid levels are negligible, consisting primarily of charged tRNAs and whatever is being produced by low-level proteolysis. Our initial experiment was to see the effect of omitting each of the amino acids individually. We found that each of the amino acids, except arginine, could be omitted from the reaction with negligible effect upon the resulting luciferase signal (Fig. 1 ). This result was obtained with lysate prepared in our laboratories and with lysates obtained commercially. Taking this result to the extreme, we found that simultaneous omission of all the amino acids, except arginine, gave $50 \%$ of the signal obtained in the presence of all of the amino acids (data not shown). This result was obtained with lysate prepared in-house as well as with lysate obtained from commercial sources.

Curious about the origin of this effect, we looked into some of the possible explanations for the ability to omit 


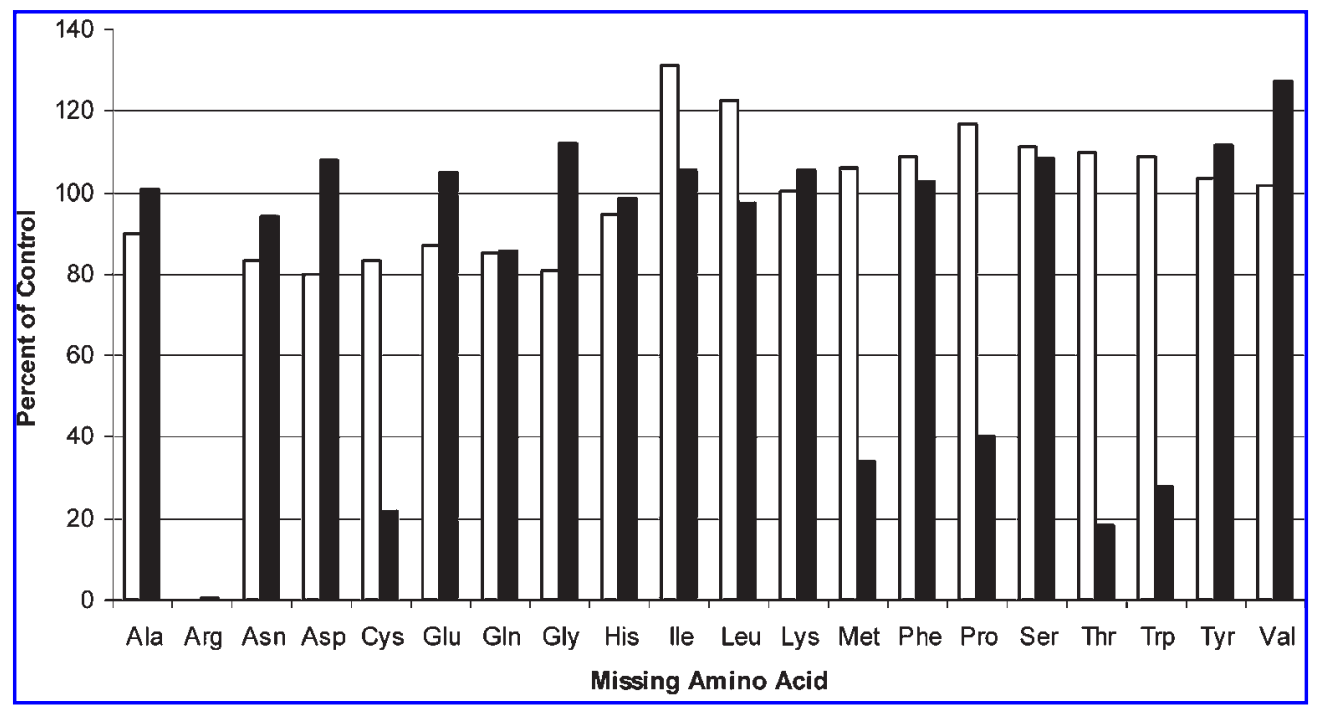

FIG. 1. Effect of omitting a single amino acid upon the production of active firefly luciferase (open columns) and $\beta$-galactosidase (filled columns) in E. coli S30 lysates. These assays were performed in 96-well format using $150 \mu M$ concentrations of each amino acid except for the one noted on the horizontal axis. The control reactions contained $150 \mu M$ concentrations of all the amino acids.

amino acids and for the essentiality of arginine. The ability to omit amino acids suggests that they are present in sufficient quantity for luciferase synthesis as free amino acids, which seems unlikely given the dialysis that the lysate undergoes, as charged tRNAs, or as a result of nonspecific proteolysis during the assay. If the amino acids were present as charged tRNAs, then tRNA synthesis inhibitors would be ineffective, and this is clearly not the case (vide infra). Our attempts to determine free amino acid concentrations in the lysate were inconclusive, leaving us with the hypothesis that a small but sufficient concentration of free amino acids, except arginine, is available during the assay, but without a ready explanation. Next, we turned our attention to arginine specifically. The concentration of arginine in E. coli cells is similar to the concentration of other amino acids; cysteine, histidine, methionine, and tryptophan are present at half or less the concentration of arginine, ${ }^{8}$ suggesting that arginine is not selectively absent from the original lysate. Arginine is a precursor to putrescine and spermidine in bacterial cells, and we considered that these compounds might make the transcription and translation process more efficient. Addition of these compounds at $100 \mu M$ to the mixture had negligible effect upon the signal in the absence or presence of arginine (data not shown). We also considered the occurrence of rare codons in luciferase, since the concentrations of certain rare tRNAs have been found to have an effect upon the rate of translation in lysates. ${ }^{9}$ The firefly luciferase gene encodes nine arginines using rare AGA or AGG codons, but they are well dispersed throughout the mRNA, and the first one occurs at codon 79. Model systems ${ }^{10,11}$ suggest that rare arginine codons distal from the start of translation that are nonconsecu- tive should have minimal effect upon translation. Finally, we decided to change reporter genes to see if this phenomenon was somehow related to luciferase. We chose lac $Z$, a gene that lacks rare arginine codons, as the reporter gene and assayed the amount of $\beta$-galactosidase produced with a standard colorimetric assay. Figure 1 shows that once again arginine is required to produce a signal. Omission of cysteine, methionine, proline, threonine, or tryptophan also results in a significantly diminished signal. This result suggests that some of the response to lowered amino acid levels is dependent upon the identity of the reporter gene, but that the absolute requirement for arginine is constant.

\section{Lowering amino acid concentration increases sensitivity to aminoacyl tRNA synthetase inhibitors}

The assay signal obtained in the presence of added arginine was sufficient to allow us to test our hypothesis that lowered amino acid concentration would sensitize the assay to tRNA synthetase inhibitors. We obtained six known inhibitors of four different tRNA synthetases and assayed them with arginine only, and with a complete amino acid mixture. Mupirocin, also known as pseudomonic acid, is a natural product that inhibits isoleucyl tRNA synthetase and is sold as a topical antibacterial..$^{12}$ Indolmycin $^{13}$ and chuangxinmycin ${ }^{14}$ are natural products that inhibit tryptophanyl tRNA synthetase. Borrelidin is a natural product that inhibits threonyl tRNA synthetase. ${ }^{15}$ Phenylalanyl tRNA synthetase inhibitors 1 and 2 (Fig. 2) are synthetic compounds derived from the results of a high-throughput screen using the purified enzyme. ${ }^{16}$ Table 1 displays the results obtained when these six inhibitors of tRNA syn- 


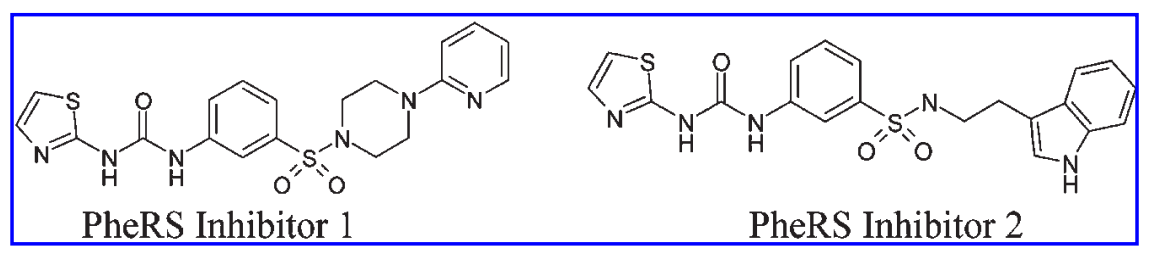

FIG. 2. Structures of phenylalanyl tRNA synthetase (PheRS) inhibitors. The characterization of these PheRS inhibitors is contained in Beyer et al. ${ }^{16}$

thetases are assayed in the absence and presence of the cognate amino acid of the appropriate tRNA synthetase. In most cases this difference is at least 16.8-fold. In contrast, known translation inhibitors that bind to the ribosome, like linezolid (Fig. 2), inhibit the assay at comparable $50 \%$ inhibitory concentration $\left(\mathrm{IC}_{50}\right)$ values regardless of the amino acid concentration. The fact that the omission of a noncognate amino acid does not alter the $\mathrm{IC}_{50}$ of an aminoacyl tRNA synthetase inhibitor and the fact that omission of any of these amino acids has no significant effect upon the $\mathrm{IC}_{50}$ of the translation inhibitor linezolid lead us to conclude that our hypothesis is correct in these particular cases, and is likely to be correct in all cases. Omission of an amino acid will sensitize the transcription and translation assay toward inhibitors of the corresponding tRNA synthetase.

\section{Omission of amino acids still produces a workable screen}

Increasing the content of the screen would be useless if the reduction in signal made the assay unsuitable for high throughput. We and others ${ }^{4}$ have used a luciferasebased screen of coupled transcription and translation in 1,536-well format. The production of luciferase in E. coli lysates is ideal for this because all of the reagents are commercially available, the amount of signal produced is reproducible, and the background signal is negligible. The key question was whether or not the reduction of signal that results from omitting all the amino acids except arginine made the assay unusable in 1,536-well format.
As can be seen from Fig. 3, the time course of the assay is essentially linear for up to $4 \mathrm{~h}$ at $30^{\circ} \mathrm{C}$, allowing us to obtain a good signal with minimal background. The use of a piezo sample distribution robot from Aurora Biosciences allowed us to spot $30 \mathrm{nl}$ per well, a DMSO concentration of $2 \%$. Figure 4 shows the results from a typical 1,536-well plate. Performed one plate at a time, the assay appears robust enough to use for screening. Unfortunately, when we tried an 18-plate run, the reproducibility across multiple plates was not good enough to run a full-file screen (data not shown). The reasons for this lack of reproducibility are not entirely clear, and the fault may be with the way the plates were spotted rather than the reproducibility of the assay. We decided that rather than determine the reason for the lack of reproducibility when all the amino acids except arginine are omitted, we would limit the number of amino acids omitted from the assay. We found that the screen works perfectly well if the number of amino acids omitted was limited to five, suggesting that a series of four screens could cover all the tRNA synthetases except that for arginine. For example, omitting only tryptophan, phenylalanine, histidine, methionine, and glycine produced an assay that was virtually indistinguishable from the assay containing a full complement of amino acids (average $Z^{\prime}$ for 945 plates was 0.69). We screened a large file of individual compounds using this assay lacking tryptophan, phenylalanine, histidine, methionine, and glycine. Our experience suggests that inhibitors of tryptophanyl, histidyl, and glycyl tRNA synthetases will be hard to find, while inhibitors of phenylalanyl and methionyl tRNA synthetases

Table 1. Addition of Cognate Amino Acid Increases the IC $_{50}$ of tRNA Synthetase Inhibitors

\begin{tabular}{|c|c|c|c|}
\hline \multirow[b]{2}{*}{ Compound } & \multicolumn{3}{|c|}{$I C_{50}(\mu M)$} \\
\hline & $\begin{array}{l}\text { Arg alone } \\
(150 \mu \mathrm{M})\end{array}$ & $\begin{array}{c}\text { All amino acids } \\
(150 \mu M \text { each })\end{array}$ & $\begin{array}{l}\text { Fold } \\
\text { change }\end{array}$ \\
\hline Mupirocin & 0.030 & 0.230 & 7.7 \\
\hline Indolmycin & 6.8 & $>286$ & $>42$ \\
\hline Chuangxinmycin & 17 & $>286$ & $>17$ \\
\hline Borrelidin & 0.007 & 0.042 & 6 \\
\hline Phenylalanyl tRNA synthetase inhibitor 1 & 0.23 & 3.9 & 17 \\
\hline Phenylalanyl tRNA synthetase inhibitor 2 & 0.050 & 7.9 & 160 \\
\hline Linezolid & 5.4 & 3.0 & $\sim 1$ \\
\hline
\end{tabular}




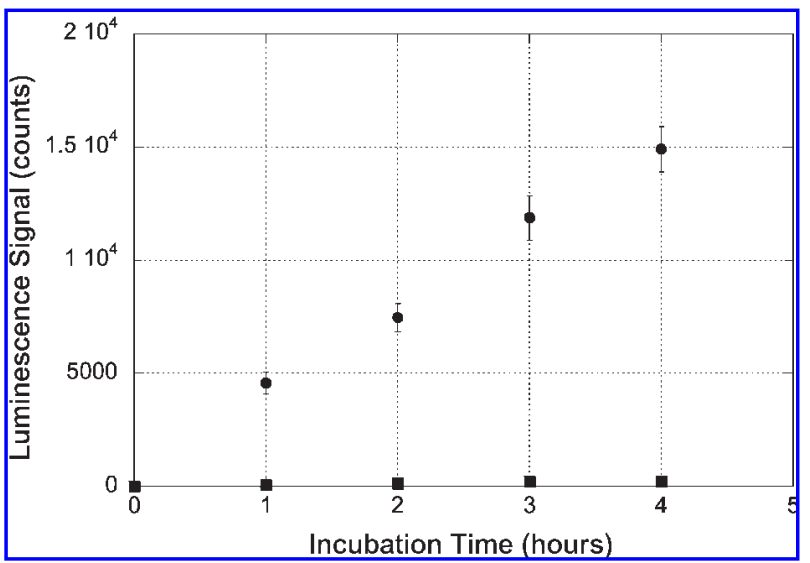

FIG. 3. Time course of the transcription and translation assay without added amino acids. This graph shows both the uninhibited signal $(\mathbf{O})$ and the background signal (ם). Each time point represents the average signal from one half of a 1,536well plate. Error bars represent \pm 1 standard deviation from the mean.

are many and varied. Specific compounds resulting from this screen and their elaboration to leads will be described in due course.

\section{The assay can be used for target identification}

We received a partially purified natural product extract for testing recently that illustrates the power of this assay system. This partially purified material had confirmed antibacterial activity towards E. coli, and had shown strong and selective inhibition of protein synthesis in macromolecular synthesis assays. ${ }^{17}$ Our first pass was to compare the extract's $\mathrm{IC}_{50}$ in the presence of only arginine to its $\mathrm{IC}_{50}$ in the presence of all amino acids. These assays were performed in 96-well format. We measured the $\mathrm{IC}_{50}$ values instead of assaying at a single concentration because it was more efficient for us to do it this way, not because an assay at a single concentration of extract would necessarily give ambiguous results. The results suggested that inhibition of a tRNA synthetase was the likely target (data not shown). Simultaneous genetic studies revealed a resistance mutation in Neisseria gonorrhoeae that mapped to a $10-\mathrm{kb}$ fragment of the genome containing the genes for phenylalanyl and threonyl tRNA synthetases. We therefore restricted our second phase of assays to these two synthetases, sparing the precious extract. $\mathrm{An}^{\mathrm{IC}_{50}}$ was determined for the extract with assay mixtures containing arginine alone, arginine with $150 \mu \mathrm{M}$ phenylalanine, and arginine with $150 \mu M$ threonine. The data (Table 2) clearly supported threonyl tRNA synthetase as the target for the natural product contained in the partially purified extract, since the addition of threonine to the assay elevates the $\mathrm{IC}_{50}$ by a factor of approximately 20 .
To demonstrate that this method would likely have worked just as well if no hints had been obtained from mutagenesis studies, we measured $\mathrm{IC}_{50}$ values with borrelidin, a known inhibitor of bacterial threonyl tRNA synthease, using assay mixtures that contained either added arginine alone or arginine plus one other amino acid. These results (Fig. 5), obtained using the 96-well format, show clearly that the only addition of threonine increases the $\mathrm{IC}_{50}$ of borrelidin, identifying threonyl tRNA synthetase as the target.

\section{Discussion}

This work exploits a unique observation to increase the utility of a classic method of screening for inhibitors of transcription and translation, and to provide a convenient method to identify which, if any, tRNA synthetase is the target of a novel antibacterial agent. We think that arginine is present in the original lysate, and that it is unlikely that arginine is selectively removed from the system by the run-off and subsequent dialysis. A requirement for arginine-derived polyamines is clearly not the reason, nor is the presence or absence of rare codons in the reporter gene. The reason for the requirement for arginine, whether luciferase or $\beta$-galactosidase is the reporter, remains unclear.

Regardless of the origin for the arginine requirement, omission of amino acids from the transcription and translation assay gives us the ability to add content to the highthroughput screen by sensitizing the assay to inhibitors of selected tRNA synthetases. Identification of a hit as a tRNA synthetase inhibitor can be made by assaying the

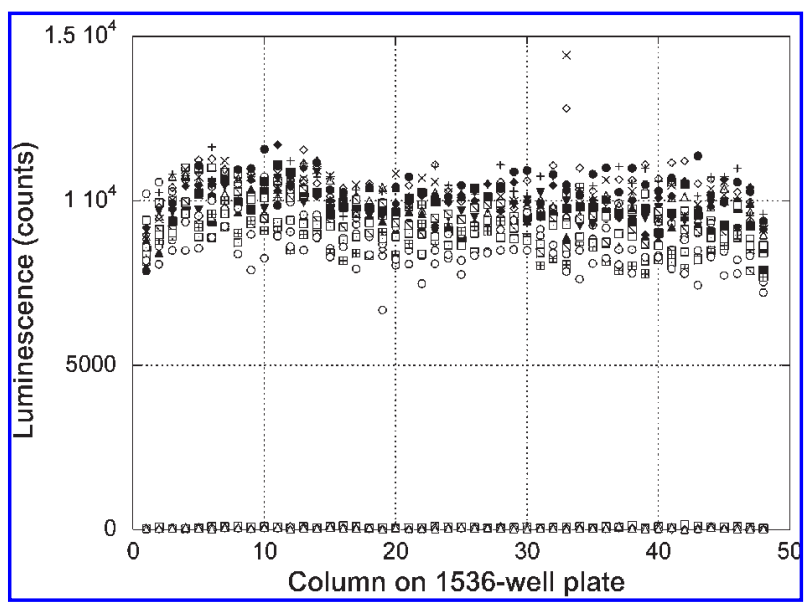

FIG. 4. Screening assay containing only arginine run in 1,536-well format. Typical results are given for a single 1,536well plate omitting all amino acids except arginine. The signal averaged 9,607 counts with a coefficient of variation of $9 \%$. The background averaged 16 counts with a standard deviation of 20 counts. The data give a $Z^{\prime}$ of 0.73 . 
Table 2. Increase of $\mathrm{IC}_{50}$ With the Addition of Threonine Suggests That the Extract InHIBITS THREONYL tRNA SynthetASE

\begin{tabular}{lc}
\hline Assay condition & $I C_{50}(\mu M)$ \\
\hline Arginine only & 1.2 \\
Arg $+150 \mu M$ Phe & 1.5 \\
Arg $+150 \mu M$ Thr & 25 \\
\hline
\end{tabular}

compound in the presence and absence of the cognate amino acid. The fold change observed for tRNA synthetase inhibitors is at least six, but may be more than two orders of magnitude (Table 1). The wide range of fold changes is consistent with the mechanisms by which these compounds inhibit their respective tRNA synthetases. Borrelidin, which shows a fold change of only 6.7, inhibits threonyl tRNA synthetases using a mechanism that has been called noncompetitive with both threonine and ATP, ${ }^{15}$ suggesting that borrelidin binds with equal affinity to the free enzyme and to the enzyme-substrate complex. Taken at the simplest level, this mechanism should not be susceptible to competition by amino acid at all. The origin of the 6.7-fold increase must therefore be a combination of error in the assay and the degree to which inhibition in our system deviates from pure noncompetitive kinetics. Mupirocin, or pseudomonic acid, which shows a fold change of only seven, inhibits isoleucyl tRNA synthetase from both Gram-positive ${ }^{18}$ and Gram-negative ${ }^{12}$ organisms using a slow, tight-binding mechanism. Inhibition proceeds through an initial binding to the enzyme, with affinity of approximately 60 $\mathrm{n} M$, which is competitive with substrate. This is followed by a slow tightening of the binding due to conformational change in the protein, leading to an overall affinity constant of approximately $20 \mathrm{pM}$. The initial phase is competitive, and therefore susceptible to competition by isoleucine. The slow-binding phase, however, is not susceptible to amino acid competition and might be expected to reduce the magnitude of the effect of isoleucine. Both indolmycin ${ }^{13}$ and chuangxinmycin ${ }^{14}$ inhibit tryptophanyl tRNA synthetase and are competitive with tryptophan. Phenylalanyl tRNA synthetase inhibitors 1 and 2 were developed from hits in a recent high-throughput screen. ${ }^{16}$ Phenylalanyl tRNA synthetase inhibitor 2 was shown to be competitive with phenylalanine, and by analogy one would expect phenylalanyl tRNA synthetase inhibitor 1 to be competitive as well. All these competitive inhibitors exhibit $\geq 17$-fold changes in $\mathrm{IC}_{50}$ in an assay supplemented with the cognate amino acid.

The use of this assay as a tool in discovering the target of novel antibacterial agents is perhaps more important than its use as a screening tool. Whole cell screening of natural product broths and of synthetic libraries remains a significant contributor to the discovery of novel antibac-

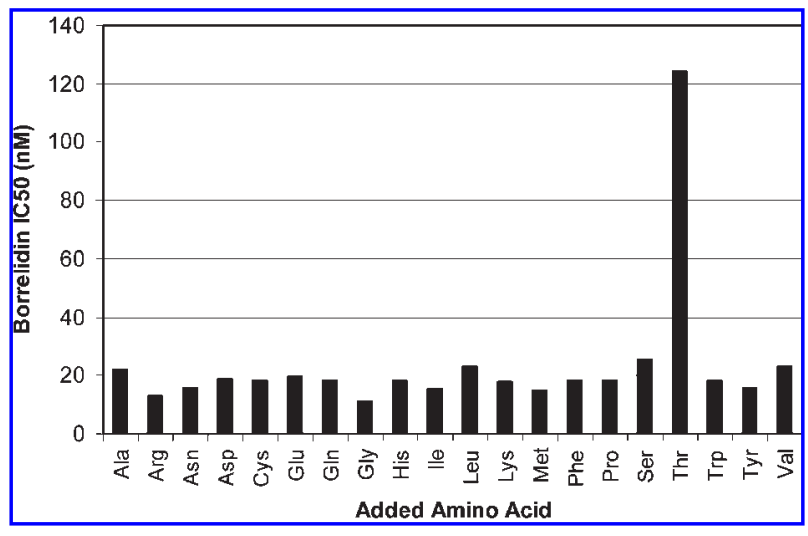

FIG. 5. Borrelidin $\mathrm{IC}_{50}$ as a function of which amino acid is added to the assay. Each bar represents the $\mathrm{IC}_{50}$ of borrelidin in the presence of $150 \mu M$ arginine plus the amino acid listed on the horizontal axis at $150 \mu M$.

terials. ${ }^{19}$ Prior to the development of this assay a tRNA synthetase inhibitor that inhibited translation in macromolecular synthesis assays, but gave modest or insignificant inhibition of coupled transcription and translation, might be discarded. As an alternative, a battery of tRNA synthetase assays could be employed, or time-consuming genetics tools might be used, as was done in the example above, to elucidate the true molecular target. These options, however, require a significant investment of time and expense. This assay, which requires only a set of commercially available reagents and a set of amino acid solutions, allows the investigator to obtain a rapid, high-confidence method to determine whether or not the novel compound inhibits a tRNA synthetase. The assay then allows the investigator to determine which tRNA synthetase is inhibited. The only tRNA synthetase that is not strictly covered by this assay is argininyl tRNA synthetase, since the requirement for arginine addition precludes comparing an $\mathrm{IC}_{50}$ in the presence and absence of amino acid.

\section{Acknowledgments}

The authors thank Annette Meyer and her team for bringing the natural product extract to us for analysis.

\section{References}

1. Mukhtar T, Wright G: Streptogramins, oxazolidinones, and other inhibitors of bacterial protein synthesis. Chem Rev 2005; 105:529-542.

2. Zubay G: In vitro synthesis of protein in microbial dystems. Annu Rev Genet 1973;7:267-281.

3. Murray R, Melchior E, Hagadorn J, Marotti K: Staphylococcus aureus cell extract transcription-translation assay: firefly luciferase system for evaluating protein translation inhibitors. Antimicrob Agents Chemother 2001;45:19001904. 
4. Kariv I, Cao H, Marvil P, Bobkova E, Bukhtiyarov Y, Yan $\mathrm{Y}$, et al.: Identification of inhibitors of bacterial transcription/translation machinery utilizing a minaturized 1536well format screen. J Biomol Screen 2001;6:233-243.

5. Hurdle J, O'Neill A, Chopra I: Prospects for aminoacyltRNA synthetase inhibitors as new antimicrobial agents. Antimicrob Agents Chemother 2005;49:4821-4833.

6. Critchley I, Young C, Stone K, Ochsner U, Guiles J, Tarasow T, et al.: Antibacterial activity of REP8839, a new antibiotic for topical use. Antimicrob Agents Chemother 2005;49:4247-4252.

7. Macarron R, Mensah L, Cid C, Carranza C, Benson N, Pope A, et al.: A homogeneous method to measure aminoacyl-tRNA synthetase aminoacylation activity using scintillation proximity assay technology. Anal Biochem 2000; 284:183-190.

8. Neidhardt FC, Umbarger HE: Chemical composition of Escherichia coli. In: Escherichia coli and Salmonella: Cellular and Molecular Biology (Neihardt FC, ed.), pp. 13-16. ASM Press, Washington, DC, 1996.

9. Jiang X, Nakano H, Kigawa T, Yabuki T, Yokoyama S, Clark D, et al.: Dosage effect of minor arginyl- and isoleucyl-tRNAs on protein synthesis in an Escherichia coli in vitro coupled transcription/translation system. $\underline{\text { J Biosci }}$ Bioeng 2001;91:53-57.

10. Varenne S, Baty D, Verheij H, Shire D, Lazdunski C: The maximum rate of gene expression is dependent in the downstream context of unfavorable codons. Biochimie 1989; 71:1221-1229.

11. Rosenberg A, Goldman E, Dunn J, Studier F, Zubay G: Effect of consecutive AGG codons on translation in Escherichia coli, demonstrated with a versatile codon test system. J Bacteriol 1993;175:716-722.

12. Hughes J, Mellows G: Interaction of pseudomonic acid A with Escherichia coli B isoleucyl-tRNA synthetase. Biochem J 1980;191:209-219.

13. Werner R, Thorpe L, Reuter W, Nierhaus K: Indolmycin inhibits prokaryotic tryptophanyl tRNA ligase. Eur J Biochem 1976;68:1-3.
14. Brown M, Carter P, Fenwick A, Fosberry A, Hamprecht $\mathrm{D}$, Hibbs $\mathrm{M}$, et al.: The antimicrobial natural product chuangxinmycin and some synthetic analogs are potent and selective inhibitors of bacterial tryptophanyl tRNA synthetase. Bioorg Med Chem Lett 2002;12:3171-3174.

15. Ruan B, Bovee M, Sacher M, Stathopoulos C, Poralla K, Francklyn $\mathrm{C}$, et al.: A unique hydrophobic cluster near the active site contributes to differences in borrelidin inhibition among threonyl tRNA synthetases. $\underline{\mathrm{J} \mathrm{Biol} \mathrm{Chem}}$ 2005;280:571-577.

16. Beyer D, Kroll H, Endermann R, Schiffer G, Siegel S, Bauser M, et al.: New class of bacterial phenylalanyltRNA synthetase inhibitors with high potency and broadspectrum activity. Antimicrob Agents Chemother 2004;48: 525-532.

17. Hughes J, Mellows G: On the mode of action of pseudamonic acid: inhibition of protein synthesis in Staphylococcus aureus. J Antibiot (Tokyo) 1978;4:330-335.

18. Pope A, Moore K, McVey M, Mensah L, Benson N, Osbourne $\mathrm{N}$, et al.: Characterization of isoleucyl-tRNA synthase from Staphylococcus aureus. II. Mechanism of inhibition by reaction intermediate and psuedamonic acid analogs studied using transient and steady-state kinetics. $J$ Biol Chem 1998;273:31691-31701.

19. Wang J, Soisson SM, Young K, Shoop W, Kodali S, Galgoti $\mathrm{A}$, et al:: Platensimycin is a selective FabF inhibitor with potent antibacterial activity. Nature 2006;441:358361

Address reprint requests to: Tod P. Holler, Ph.D. Pfizer Global Research and Development Michigan Laboratories 2800 Plymouth Road Ann Arbor, MI 48105

E-mail: tod.holler@pfizer.com 

This article has been cited by:

1. Letizia Brandi, Attilio Fabbretti, Cynthia L Pon, Albert E Dahlberg, Claudio O Gualerzi. 2008. Initiation of protein synthesis: a target for antimicrobials. Expert Opinion on Therapeutic Targets 12:5, 519-534. [CrossRef] 